\title{
Magnetic critical behavior of two-dimensional random-bond Potts ferromagnets in confined geometries
}

\author{
Christophe Chatelain and Bertrand Berchet \\ Laboratoire de Physique des Matériaux $\doteq$ Université Henri Poincaré, \\ Nancy 1, BP 239, F-54506 Vandouvre les Nancy Cedex, France
}

(23 March 1999)

\begin{abstract}
We present a numerical study of $2 D$ random-bond Potts ferromagnets. The model is studied both below and above the critical value $Q_{c}=4$ which discriminates between second and first-order transitions in the pure system. Two geometries are considered, namely cylinders and square-shaped systems, and the critical behavior is investigated through conformal invariance techniques which were recently shown to be valid, even in the randomness-induced second-order phase transition regime $Q>4$. In the cylinder geometry, connectivity transfer matrix calculations provide a simple test to find the range of disorder amplitudes which is characteristic of the disordered fixed point. The scaling dimensions then follow from the exponential decay of correlations along the strip. Monte Carlo simulations of spin systems on the other hand are generally performed on systems of rectangular shape on the square lattice, but the data are then perturbed by strong surface effects. The conformal mapping of a semi-infinite system inside a square enables us to take into account boundary effects explicitly and leads to an accurate determination of the scaling dimensions. The techniques are applied to different values of $Q$ in the range 3-64.
\end{abstract}

\section{INTRODUCTION}

The presence of impurities can have significative effects on the nature of phase transitions. Both from experimental and theoretical perspectives, the study of the influence of randomness is of great importance. Experimental evidences of the effect of random quenched impurities in two-dimensional systems were found in order-disorder phase transitions of adsorbed atomic layers belonging, in the pure case, to the $Q=4$-state Potts model universality class [1,2]. In the presence of disorder, the critical exponents are modified. On the other hand, no modification is found when the pure system belongs to the Ising universality class [3].

The study of disordered systems is a quite active field of research in statistical physics, and the resort to largescale Monte Carlo simulations is often helpful [4]. Numerical investigations of the critical properties of random systems require averages over disorder realizations. Standard techniques, like Finite-Size Scaling (hereafter referred to as FSS) or temperature dependence of the physical quantities were extensively used, and, more recently, conformal invariance techniques were shown to provide accurate results.

The effect of quenched bond randomness in a system which undergoes a second-order phase transition in the homogeneous case has been considered first. It is well understood since Harris proposed a relevance criterion for the case of fluctuating interactions [5]. Disorder appears to be a relevant perturbation when the specific heat exponent $\alpha$ of the pure system is positive. Since in the two-dimensional Ising model (IM) $\alpha$ vanishes due to the logarithmic Onsager singularity, this model was carefully studied in the 1980s [6]. The analogous situation when the pure system exhibits a first-order transition was less well studied, in spite of the early work of Imry and Wortis who argued that quenched disorder could induce a second-order phase transition [7]. This argument was then rigorously proved by Aizenman and Wehr, and Hui and Berker [8,9]. In two dimensions, even an infinitesimal amount of quenched impurities changes the transition into a continuous one.

The first intensive Monte Carlo (MC) study of the effect of disorder at a first-order phase transition is due to Chen, Ferrenberg and Landau. These authors studied the $Q=8$-state two-dimensional random-bond Potts model (RBPM), which, in the pure case, is known to exhibit a first-order phase transition when $Q>4$, the larger the value of $Q$, the sharper the transition [10]. Taking advantage of duality, they performed a finite-size scaling study at the critical point of a self-dual disordered system 11, 12 and definitively showed that the transition becomes of second order in the presence of bond randomness. Their results, together with other related works 13 16], suggested that any two-dimensional random system should belong to the 2D pure IM universality class. These results were also coherent with real experiments [1].

In recent papers, Cardy and Jacobsen used a different approach 17, 18], based on the connectivity transfer matrix (TM) formalism of Blöte and Nightingale [19]. They studied random-bond Potts models for different values of $Q$ and with a bimodal probability distribution of coupling strengths. Their estimations of the critical ex- 
ponents lead to a continuous variation of $\beta / \nu$ with $Q$. This result is in accordance with previous theoretical calculations and MC simulations when $Q \leq 4$ [20.21. In the randomness-induced second-order phase transition regime $Q>4, \beta / \nu$ is quite different from the Ising value of $\frac{1}{8}$ and particularly in sharp disagreement with the Monte Carlo results of Ref. [12] for $Q=8$. Since then, Monte Carlo simulations were performed by different groups at $Q=8$ 22 24]. The choice of the value $Q=8$ was motivated by the value of the correlation length in the pure case $(\xi=23.87$ in lattice spacing units) 25]. MC simulations which enable to discriminate between a first-order regime and a second-order transition can indeed be performed easily with systems of larger sizes. These studies led to partially conflicting results given in Table If, but they eventually found an explanation in terms of a crossover behavior in a recent work of Picco [24. While theoretical calculations are generally managed in the weak disorder regime (perturbation expansion around the homogeneous system fixed point), the range of disorder amplitude must be chosen carefully in numerical studies, since the random fixed point (FP) can be perturbed by crossover effects due to the pure and/or the percolation unstable fixed points. The disordered FP properties are thus more easily observed with strong randomness. A disorder amplitude $r$, given by the ratio of the two types of couplings (distributed according to a binary distribution), in the range 8-20 appears to be adapted to a numerical analysis and gives a good estimate of the disordered fixed point exponents [24.26] as already observed in the $2 D$ random-bond Ising model (RBIM) 27,28.

TABLE I. Bulk magnetic scaling index obtained by different groups in the 8-state Potts model.

\begin{tabular}{llll}
\hline \hline Authors & $r$ & $\beta / \nu$ & Technique \\
\hline Chen et al, Ref. 12] & 2 & $0.118(2)$ & $\mathrm{MC}$ \\
Cardy and Jacobsen, Ref. 17] & 2 & $0.142(4)$ & TM \\
Chatelain and Berche, Ref. [22] & 10 & $0.153(3)$ & $\mathrm{MC}$ \\
Picco, Ref. 24] & 10 & $0.153(1)$ & $\mathrm{MC}$ \\
\hline \hline
\end{tabular}

The surface properties of dilute or random-bond magnetic systems were paid less attention. The whole set of bulk and surface critical exponents of a given system is determined by the anomalous dimensions of the relevant scaling fields which enter the homogeneity assumption of the singular free energies 29]. The $(1,1)$ surface of the disordered Ising model on a square lattice has only recently been investigated through $\mathrm{MC}$ simulations by Selke et al. [30]. The critical exponent $\beta_{1}$ of the boundary magnetization was found to be equal within error bars to its value in the pure 2D IM. The surface properties of the 8 -state RBPM were also computed in Ref. [22].

In this paper, we are interested in the bulk critical behavior of disordered Potts ferromagnets, and in the evolution of their properties as the number of states $Q$ increases. The Hamiltonian of the model is

$$
-\beta \mathcal{H}=\sum_{(i j)} K_{i j} \delta_{\sigma_{i}, \sigma_{j}}
$$

where the spins can take $Q$ different values and the coupling strengths between nearest neighbor spins are taken from a binary probability distribution

$$
\mathcal{P}\left(K_{i j}\right)=p \delta\left(K_{i j}-r K\right)+(1-p) \delta\left(K_{i j}-K\right)
$$

with $p=1 / 2$, which guarantees the self-duality relation

$$
\left(e^{r K_{c}}-1\right)\left(e^{K_{c}}-1\right)=Q .
$$

The value $r=1$ corresponds to the pure model and $r \rightarrow \infty$ to the percolation limit.

In the present work, following previous studies, we use the powerful methods of conformal invariance. Talapov et al. studied numerically the critical-point correlation functions in the 2D RBIM on the torus [31] and took into account the finite-size effects through a convenient conformal rescaling [32,33]. In the cylinder geometry, conformal invariance methods have also been successfully applied. In the two-dimensional RBIM, randomness being a marginally irrelevant perturbation, many results have been obtained via these techniques: Conformal anomaly, correlation decay, gap-exponent relation for long strips 34 36]. At randomness-induced second-order phase transitions, conformal techniques have also been used already [17, 18, 37 and numerical evidences for the validity of the conformal covariance assumption for correlation functions and density profiles were recently reported [38]. It is well known that in disordered spin systems, the strong fluctuations of couplings from sample to sample require careful averaging procedures 39 41]. For that reason, the study of the probability distributions must be performed in order to guarantee that the average quantities, which should obey the conformal covariance assumption, are correctly obtained numerically. A comparison between grand canonical disorder (GCD) and canonical disorder (CD) will also be given.

The plan of the paper is the following: In Sec II, we present the results of connectivity transfer matrix calculations on strips with periodic boundary conditions for different values of $Q$. The order parameter correlation function, after disorder average, leads to estimates of the magnetic scaling index for different strip sizes. From our knowledge in the case $Q=8$ [38], it appears that these computations are suitable for the determination of a convenient disorder amplitude in order to reach the disordered FP. At large disorder amplitudes $(r \simeq 10)$, the behavior of the effective central charge can indeed discriminate between random and percolation fixed points. In Sec. III, we report Monte Carlo simulations in a square geometry with the above-mentioned disorder amplitude. The magnetization correlation function and density profile give access to refined values for the corresponding exponents. A discussion of the results is given in Section IV. Attention is paid to take into account the different sources of error for the results reported in this work. 


\section{CYLINDER GEOMETRY AND DISORDERED FIXED POINT}

\section{A. Free energy and central charge}

In the strip geometry, we used the Blöte and Nightingale connectivity transfer matrix method 19. In disordered systems, transfer operators in the time direction do not commute and, as a consequence, the free energy density is no longer defined by the largest eigenvalue of a single TM, but in terms of the leading Lyapunov exponent. For a strip of size $L$ with periodic boundary conditions, the leading Lyapunov exponent follows from the Furstenberg method 42:

$$
\begin{aligned}
\Lambda_{0}(L) & =\lim _{m \rightarrow \infty} \frac{1}{m} \ln \|\left(\prod_{k=1}^{m} \mathbf{T}_{k}\right)\left|v_{0}\right\rangle \| \\
& =\lim _{m \rightarrow \infty} \Lambda_{0}(L ; m),
\end{aligned}
$$

where $\mathbf{T}_{k}$ is the transfer matrix between columns $k-1$ and $k$ and $\left|v_{0}\right\rangle$ is a suitable unit initial vector. The free energy density is thus given by

$$
\left[f_{0}(L)\right]_{\mathrm{av}}=-L^{-1} \Lambda_{0}(L),
$$

where $[\ldots]_{\text {av }}$ denotes the average over disorder realizations.

In the following, we considered canonical disorder, a situation in which exactly the same numbers of couplings $K$ and $r K$ are distributed over the bonds of the whole system of length $\sim 10^{6}$. This choice contributes to reduce sample fluctuations. This is shown in Fig. 11 where the stability of the free energy density is compared to the standard grand canonical disorder for different runs up to $m=10^{6}$ iterations of the TM.

In Eq. (4), the disorder average is implicitly performed through an infinite number of iterations of the transfer matrix. In our computations, only a finite number $m$ is used, leading to approximate values denoted by $\Lambda_{0}^{(i)}(L ; m)$ for different runs labelled by an integer $i=1, M$. The leading Lyapunov exponent and the corresponding eigenvector, $\left|\Lambda_{0}\right\rangle$, obtained after $m=10^{6}$ iterations of the TM, are then averaged over $M=48$ independent runs. The average free energy density of Eq. (5) is thus replaced, in the calculations, by

$$
\begin{aligned}
{\left[f_{0}(L)\right]_{\mathrm{av}} } & \simeq\left[f_{0}(L)\right]_{M} \\
& =-L^{-1}\left(\frac{1}{M} \sum_{i=1}^{M} \Lambda_{0}^{(i)}\left(L ; 10^{6}\right)\right) .
\end{aligned}
$$

The value $M=48$ was chosen in order to guarantee a stability of the averaged quantities with a relative error better than $10^{-5}$ for the free energy density and than $4 \times 10^{-5}$ for the components of the corresponding eigenvector. The computations are then performed on strips of sizes $L=2$ to 8 .

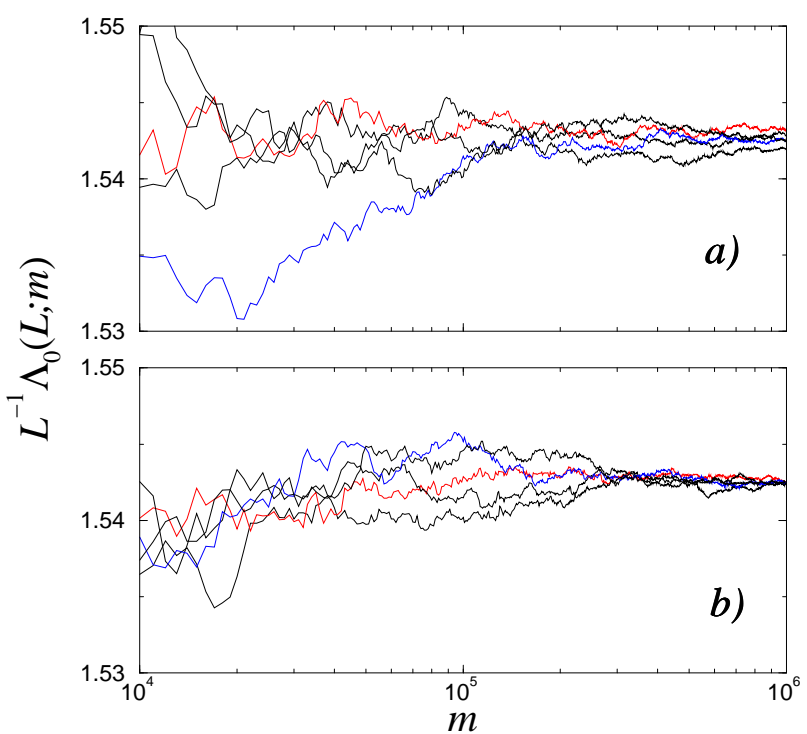

FIG. 1. Free energy density (up to an additive constant $\ln Q$ ) vs $m$, the number of iterations of the TM for a strip of size $L=6(Q=8, r=10)$ with 5 realizations in grand canonical (a) and canonical (b) disorder.

The numerical investigation of critical properties in random systems requires the knowledge of the range of disorder amplitude (measured here by the ratio $r$ between strong and weak couplings) for which the fixed point properties is reached. Outside this regime, strong crossover effects perturb the data 38]. A convenient disorder amplitude $r$ can be obtained from the behavior of the effective central charge, which increases when the system approaches the disordered fixed point in non-unitary theories as it seems to be the case in the RBPM 18,43. The central charge $c$ is defined by the leading size dependence of the free energy density, and, since the strip sizes are quite small, corrections to scaling must be included:

$$
\left[f_{0}(L)\right]_{\mathrm{av}}=f_{\mathrm{reg}}-\frac{\pi c}{6} L^{-2}+A L^{-4}
$$

The comparison between successive sizes $L$ and $L+l$ allows us to define a reduced difference which leads to

$$
\begin{aligned}
{\left[\Delta f_{l}(L)\right]_{\mathrm{av}} } & \equiv \frac{6}{\pi} \frac{\left[f_{0}(L)\right]_{\mathrm{av}}-\left[f_{0}(L+l)\right]_{\mathrm{av}}}{(L+l)^{-2}-L^{-2}} \\
& =c-\frac{6}{\pi} A \lambda,
\end{aligned}
$$

where the reduced parameter $\lambda$ is given by

$$
\lambda=\frac{(L+l)^{-4}-L^{-4}}{(L+l)^{-2}-L^{-2}} .
$$

In the thermodynamic limit, the central charge $c$ then follows from a linear fit as shown in Fig 2 for strips of sizes $L=2$ to 8 in the case $Q=3$. We restricted our study to integer values of $r$ and the data for the effective central charge at different disorder amplitudes are given 
in the Table [1]. We observe that the value of $c$ is strongly depending on the disorder amplitude: It increases from the weak disorder limit up to a maximum value and then decreases slowly as $r$ increases.

The central charge at the random fixed point (i.e. the maximal value obtained for an optimal disorder amplitude $\left.r^{\star}(Q)\right)$ is shown in Fig. 3. Assuming a linear behavior in $\ln Q$ 37] which preserves the Ising value $c(Q=2)=1 / 2[18]$, one gets

$$
c(Q)=\frac{\ln Q}{2 \ln 2}
$$

whilst the percolation limit leads to $c(Q)=\frac{5 \sqrt{3}}{4 \pi} \ln Q[18]$. The two behaviors are shown in Fig. 3. The numerical data are in good agreement with Eq. 10 and are accurate enough to consider that the random $\mathrm{FP}$ has been reached at $r^{\star}$ (whose values are coherent with those found by Jacobsen and Cardy [18]: $r^{\star}(3)=7, r^{\star}(8)=9$ and $\left.r^{\star}(64)=10\right)$. In the following, the scaling properties will be studied at the optimal disorder amplitudes in contradistinction with previous papers [17, 18].

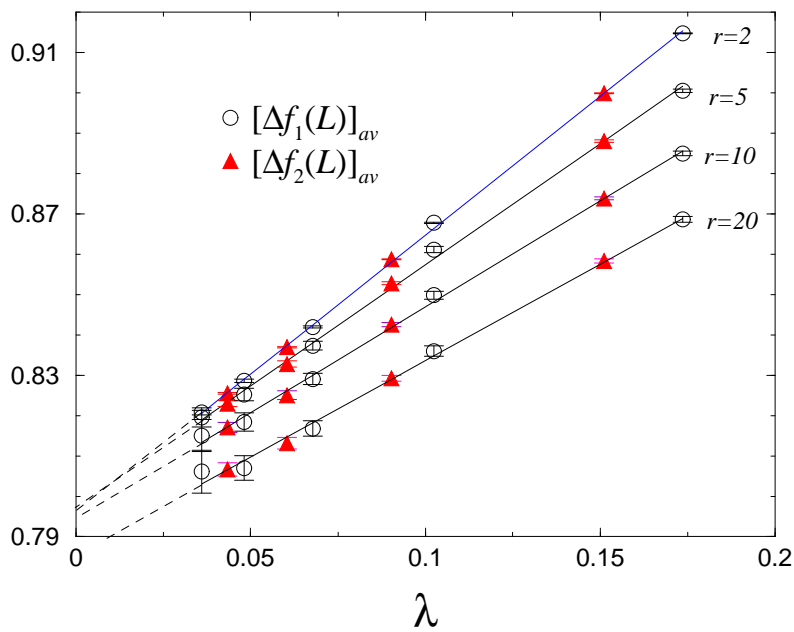

FIG. 2. Reduced difference between the free energies at different sizes $\left[\Delta f_{l}(L)\right]_{\text {av }}$ for different values of the disorder amplitude $r(Q=3)$. The central charge is given by the intercept via a linear fit. The parameter $l$ is defined in Eq. (8).

TABLE II. Extrapolation of the effective central charge $c$ in the thermodynamic limit for the different values of $Q$ and $r$. At each $Q$, the larger values of $c$ (written in bold face) correspond to the random fixed point regime with an optimal disorder amplitude $r^{\star}$. This maximum is not always located at the same $r$, as shown in the case $Q=3$ and 64 for two different runs.

\begin{tabular}{|c|c|c|c|c|c|c|c|c|c|}
\hline & \multicolumn{9}{|c|}{ Effective central charge at $Q=3$} \\
\hline \multirow{5}{*}{\begin{tabular}{l|}
$r$ \\
$c$ \\
$\Delta c$ \\
$c$ \\
$\Delta c$
\end{tabular}} & 2 & 4 & 5 & 6 & 7 & 10 & 20 & & \\
\hline & 0.7970 & 0.7998 & 0.79984 & 0.79969 & 0.7992 & 0.7970 & 0.7879 & & \\
\hline & $4 \times 10^{-4}$ & $4 \times 10^{-4}$ & $3.8 \times 10^{-4}$ & $3.8 \times 10^{-4}$ & $4 \times 10^{-4}$ & $4 \times 10^{-4}$ & $4 \times 10^{-4}$ & & \\
\hline & & & 0.8005 & 0.80070 & 0.80099 & & & & \\
\hline & & & $4 \times 10^{-4}$ & $3.8 \times 10^{-4}$ & $3.7 \times 10^{-4}$ & & & & \\
\hline \multirow{5}{*}{$\begin{array}{l}r \\
c \\
\Delta c \\
\end{array}$} & \multicolumn{9}{|c|}{ Effective central charge at $Q=4$} \\
\hline & 2 & 5 & 6 & 7 & 8 & 10 & 20 & & \\
\hline & 1.0043 & 1.0144 & 1.01495 & 1.01483 & 1.0142 & 1.0123 & 0.9996 & & \\
\hline & $4 \times 10^{-4}$ & $4 \times 10^{-4}$ & $4.3 \times 10^{-4}$ & $4.3 \times 10^{-4}$ & $4 \times 10^{-4}$ & $4 \times 10^{-4}$ & $4 \times 10^{-4}$ & & \\
\hline & \multicolumn{9}{|c|}{ Effective central charge at $Q=5$} \\
\hline$r$ & 2 & 5 & 6 & 7 & 8 & 10 & 20 & & \\
\hline$c$ & 1.1579 & 1.1794 & 1.1810 & 1.181593 & 1.181326 & 1.1794 & 1.1642 & & \\
\hline \multirow[t]{2}{*}{$\Delta c$} & $5 \times 10^{-4}$ & $5 \times 10^{-4}$ & $5 \times 10^{-4}$ & $4.6 \times 10^{-4}$ & $4.6 \times 10^{-4}$ & $5 \times 10^{-4}$ & $5 \times 10^{-4}$ & & \\
\hline & \multicolumn{9}{|c|}{ Effective central charge at $Q=6$} \\
\hline$r$ & 2 & 5 & 7 & 8 & 9 & 10 & 20 & & \\
\hline$c$ & 1.2764 & 1.3128 & 1.3172 & 1.3174 & 1.3168 & 1.3157 & 1.2986 & & \\
\hline \multirow[t]{2}{*}{$\Delta c$} & $5 \times 10^{-4}$ & $5 \times 10^{-4}$ & $5 \times 10^{-4}$ & $5 \times 10^{-4}$ & $5 \times 10^{-4}$ & $5 \times 10^{-4}$ & $5 \times 10^{-4}$ & & \\
\hline & \multicolumn{9}{|c|}{ Effective central charge at $Q=8$} \\
\hline$r$ & 2 & 5 & 9 & 10 & 11 & 12 & 20 & & \\
\hline$c$ & 1.4468 & 1.5203 & 1.5329 & 1.5300 & 1.5287 & 1.5270 & 1.5104 & & \\
\hline \multirow[t]{2}{*}{$\Delta c$} & $5 \times 10^{-4}$ & $5 \times 10^{-4}$ & $5 \times 10^{-4}$ & $5 \times 10^{-4}$ & $5 \times 10^{-4}$ & $5 \times 10^{-4}$ & $5 \times 10^{-4}$ & & \\
\hline & \multicolumn{9}{|c|}{ Effective central charge at $Q=15$} \\
\hline$r$ & 2 & 5 & 9 & 10 & 11 & 12 & 13 & 15 & 20 \\
\hline$c$ & 1.7313 & 1.9606 & 1.9963 & 1.9937 & 1.9930 & 1.9915 & 1.9895 & 1.9846 & 1.9708 \\
\hline \multirow[t]{2}{*}{$\Delta c$} & $6 \times 10^{-4}$ & $6 \times 10^{-4}$ & $6 \times 10^{-4}$ & $6 \times 10^{-4}$ & $6 \times 10^{-4}$ & $6 \times 10^{-4}$ & $6 \times 10^{-4}$ & $6 \times 10^{-4}$ & $6 \times 10^{-4}$ \\
\hline & \multicolumn{9}{|c|}{ Effective central charge at $Q=64$} \\
\hline$r$ & 2 & 5 & 10 & 11 & 12 & 13 & 15 & 20 & \\
\hline$c$ & 2.0302 & 2.9351 & 3.0414 & 3.0432 & 3.0430 & 3.0415 & 3.0362 & 3.0182 & \\
\hline$\Delta c$ & $7 \times 10^{-4}$ & $7 \times 10^{-4}$ & $7 \times 10^{-4}$ & $7 \times 10^{-4}$ & $7 \times 10^{-4}$ & $7 \times 10^{-4}$ & $7 \times 10^{-4}$ & $7 \times 10^{-4}$ & \\
\hline$c$ & & & & 3.0526 & 3.0528 & 3.0516 & & & \\
\hline
\end{tabular}




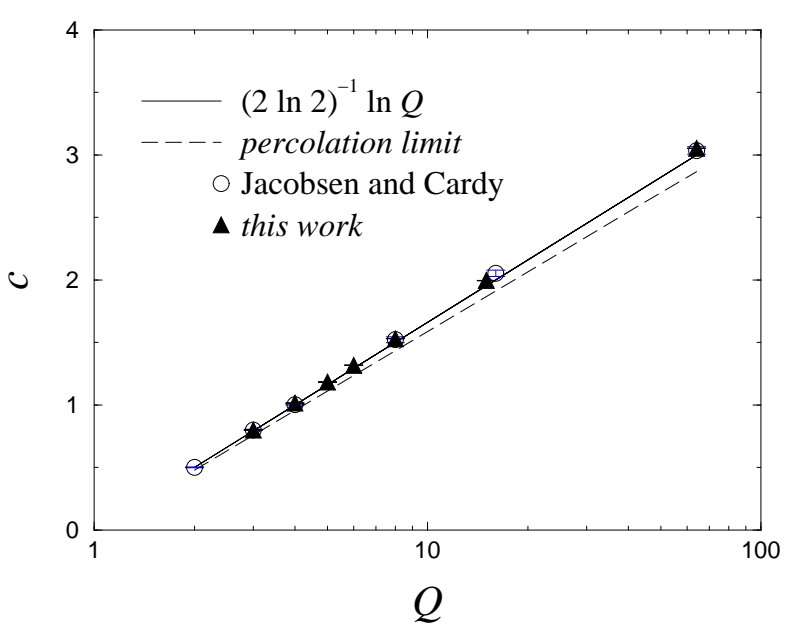

FIG. 3. Central charge at the random fixed point as a function of the number of states. The full line corresponds to $c(Q)=\ln Q /(2 \ln 2)$ while the dashed line is the percolation limit $c(Q)=5 \sqrt{3} \ln Q / 4 \pi$. Error bars are smaller than the sizes of the symbols.

\section{B. Probability distribution of the correlation function}

For a specific disorder realization, the spin-spin correlation function along the strip

$$
\left\langle G_{\sigma}(u)\right\rangle=\frac{Q\left\langle\delta_{\sigma_{j} \sigma_{j+u}}\right\rangle-1}{Q-1},
$$

where $\langle\ldots\rangle$ denotes the thermal average, is given by the probability that the spins along some row, at columns $j$ and $j+u$, are in the same state $(j$ and $j+u$ measure the position in the longitudinal direction of the strip):

$$
\left\langle\delta_{\sigma_{j} \sigma_{j+u}}\right\rangle=\frac{\left\langle\Lambda_{0}\left|\mathbf{g}_{j}\left(\prod_{k=j}^{j+u-1} \mathbf{T}_{k}^{\prime}\right) \mathbf{d}_{j+u}\right| \Lambda_{0}\right\rangle}{\left\langle\Lambda_{0}\left|\prod_{k=j}^{j+u-1} \mathbf{T}_{k}\right| \Lambda_{0}\right\rangle},
$$

where $\left|\Lambda_{0}\right\rangle$ is the ground state eigenvector and $\mathbf{T}_{k}^{\prime}$ is the transfer matrix in the extended Hilbert space which includes the connectivity with the origin site $j$. The operator $\mathbf{g}_{j}$ identifies the cluster containing $\sigma_{j}$, while $\mathbf{d}_{j+u}$ gives the appropriate weight depending on whether or not $\sigma_{j+u}$ is in the same state as $\sigma_{j}$. The computation is performed with a grand canonical disorder.

An analysis of the correlation function probability distribution is needed in order to ensure that self-averaging problems do not alter the mean values 44]. The methodology that we propose is to deduce the critical behavior from the decay of the correlation functions using conformal symmetry. Since conformal covariance assumption is supposed to be satisfied by average quantities, i.e. $\left[\left\langle G_{\sigma}(u)\right\rangle\right]_{\mathrm{av}}$, our first aim is to show that, in spite of the lack of self-averaging, our numerical experiments lead to well-defined averages.

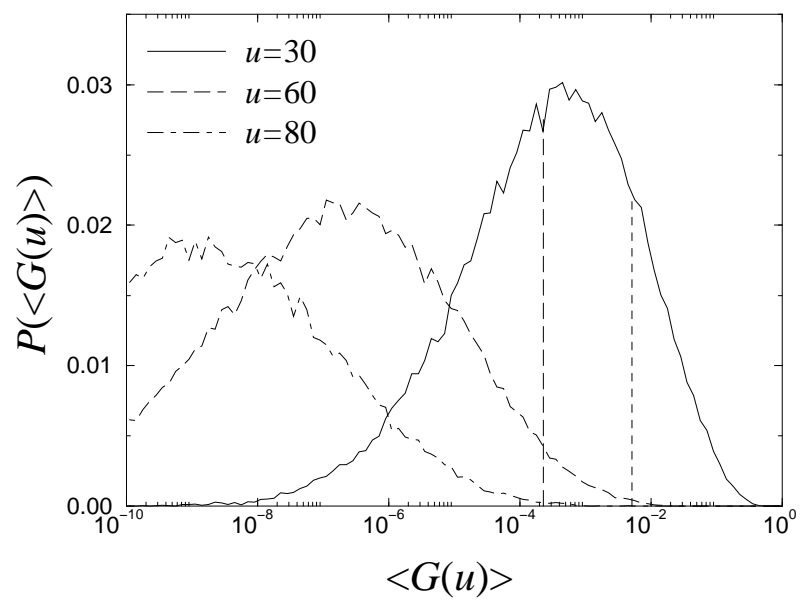

FIG. 4. Probability distribution of the correlation function after 63436 realizations of disorder for a strip of size $L=6$ $(Q=8, r=10)$. The vertical dotted line shows the average value $\left[\left\langle G_{\sigma}(30)\right\rangle\right]_{\mathrm{av}}$ while the long-dashed line shows the typical value $\mathrm{e}^{\left[\left\langle\ln G_{\sigma}(30)\right\rangle\right]_{\mathrm{av}}}$.

The probability distribution of the correlation function, as shown in Fig. 4, enables us to determine the most probable (or typical) value $G_{\sigma}^{m p}(u)$ and the average correlation function $\left[\left\langle G_{\sigma}(u)\right\rangle\right]_{\mathrm{av}}$, as well as the averaged logarithm, $\left[\ln \left\langle G_{\sigma}(u)\right\rangle\right]_{\mathrm{av}}$ at any value of the distance $u$. Compatible behaviors are found for $G_{\sigma}^{m p}(u)$ and $e^{\left[\ln \left\langle G_{\sigma}(u)\right\rangle\right]_{\mathrm{av}}}$. It is a confirmation of the essentially lognormal character of the probability distribution [44], as argued by Cardy and Jacobsen [17]. It is thus necessary to perform averages over larger numbers of samples for $\left[\left\langle G_{\sigma}(u)\right\rangle\right]_{\mathrm{av}}$ than for $\left[\ln \left\langle G_{\sigma}(u)\right\rangle\right]_{\mathrm{av}}$ to get the same relative errors.

Following Cardy and Jacobsen, since the moments of the logarithm of the correlation function are selfaveraging, a cumulant expansion can then be performed to reconstruct $\left[\left\langle G_{\sigma}(u)\right\rangle\right]_{\mathrm{av}}$ and to compare to the values obtained by averaging directly over the samples. 


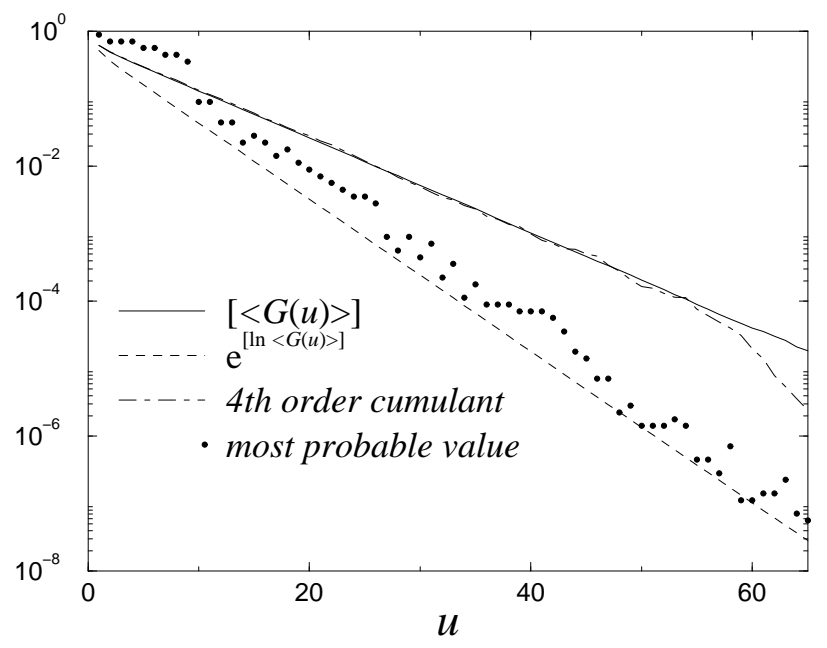

FIG. 5. Average correlation function, most probable (or typical) value and sum up to the 4 th order of the cumulant expansion obtained from 63436 realizations of disorder for a strip of size $L=6(Q=8, r=10)$.

The results in Fig. 5 (for $Q=8$ ), strengthen the credibility of the direct average, and also clearly show that the cumulant expansion up to fourth order still strongly fluctuates at large distances compared to $\left[\left\langle G_{\sigma}(u)\right\rangle\right]_{\mathrm{av}}$. In the following we will thus favour the direct averaging process, using a large number of disorder realizations.

\section{Bulk magnetic scaling dimension}

We will now use the results that follow from the assumption of conformal covariance of the average correlation functions. In the infinite complex plane $z=x+\mathrm{i} y$ (denoted by the index $\infty$ ) the correlation function exhibits the usual algebraic decay at the critical point

$$
\begin{aligned}
{\left[\left\langle G_{\sigma}(R)\right\rangle\right]_{\mathrm{av}} } & \equiv\left[\left\langle\sigma\left(z_{1}\right) \sigma\left(z_{2}\right)\right\rangle_{\infty}\right]_{\mathrm{av}} \\
& =\mathrm{const} \times R^{-2 x_{\sigma}^{b}}
\end{aligned}
$$

where $R=\left|z_{1}-z_{2}\right|$ and $x_{\sigma}^{b}=\beta / \nu$ is the bulk magnetic scaling dimension. Under a conformal mapping $w(z)$, the correlation functions of a conformally invariant 2Dsystem transforms into the new geometry according to

$$
G_{\sigma}\left(w_{1}, w_{2}\right)=\left|w^{\prime}\left(z_{1}\right)\right|^{-x_{\sigma}^{b}}\left|w^{\prime}\left(z_{2}\right)\right|^{-x_{\sigma}^{b}} G_{\sigma}\left(z_{1}, z_{2}\right) .
$$

The logarithmic tranformation $w=\frac{L}{2 \pi} \ln z$ is known to map the $z$ plane onto an infinite strip (denoted by the index st) $w=u+\mathrm{i} v$ of width $L$ with periodic boundary conditions in the transverse direction. Applying Eq. (14) in the random system where $\left[\left\langle G_{\sigma}\left(w_{1}, w_{2}\right)\right\rangle\right]_{\mathrm{av}} \equiv$ $\left[\left\langle\sigma\left(w_{1}\right) \sigma\left(w_{2}\right)\right\rangle_{\text {st }}\right]_{\text {av }}$ corresponds to the strip geometry, one gets the usual exponential decay along the strip

$$
\left[\left\langle G_{\sigma}(u)\right\rangle\right]_{\mathrm{av}}=\text { const } \times \exp \left(-\frac{2 \pi}{L} x_{\sigma}^{b} u\right),
$$

where $u=\operatorname{Re}\left(w_{2}-w_{1}\right)$. The scaling index $x_{\sigma}^{b}$ can thus be deduced from a linear fit in a semilog plot.

For each strip size $(L=2-8)$, we realized $80 \times 10^{3}$ disorder configurations. It allowed us to define mean values and error bars for the correlation functions at any point in the range $u=1-100$, taking into account the standard deviation over the samples. The non self-averaging behavior of the correlation functions induces large variances (The reduced variance $R_{X}(L) \equiv$ $\left(\left[X^{2}\right]_{\mathrm{av}}-[X]_{\mathrm{av}}^{2}\right) /[X]_{\text {av }}^{2}$ does not behave as a power law, but evolves towards a constant value when the strip size increases, e.g. $R_{G_{\sigma}(20)}(L) \rightarrow 1.50$, as already observed for several quantities by Wiseman and Domany in Refs. 40,41.). The exponents follow from an exponential fit in the range $u>5$ and $\left[\left\langle G_{\sigma}(u)\right\rangle\right]_{\text {av }}>\epsilon$, where the cutoff $\epsilon$ is introduced in order to avoid tiny numbers whose values are lower than the fluctuations. The error bars given for the exponents take into account the uncertainties of data for the correlation functions 445. The resulting values for each strip size are plotted against $L^{-1}$ which allows an extrapolation in the thermodynamic limit. This is shown in Fig. 6 in the case $Q=8$. This figure provides a confirmation of the effect of a too weak disorder: Strong crossover effects take place which lead to a wrong determination of the critical behavior with the strip sizes used here. On the other hand, at the optimal value $r^{\star}(Q)$, the exponent converges in the $L \rightarrow \infty$ limit towards a well defined final estimate.

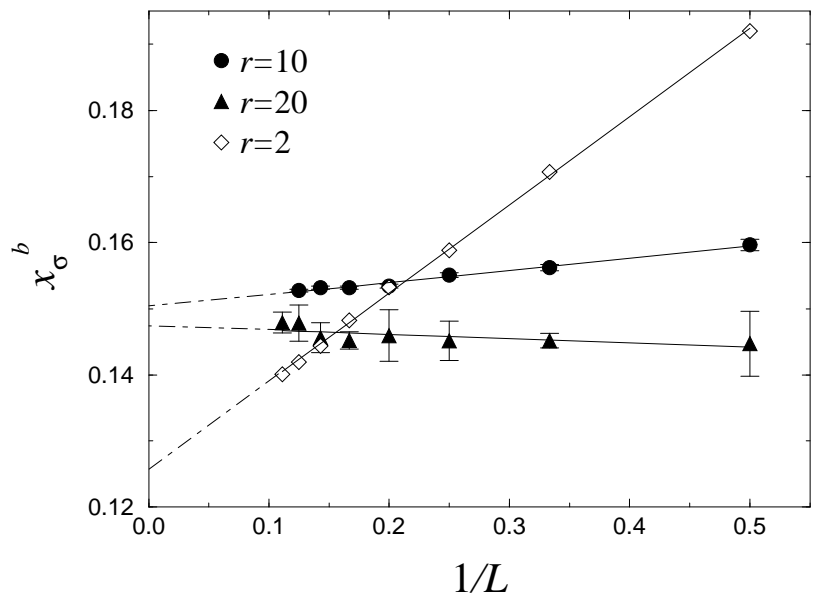

FIG. 6. Magnetic scaling index deduced from the algebraic decay of the average correlation function along the strip of size $L$ as a function of $L^{-1}$ and extrapolation in the thermodynamic limit $(Q=8, L=2-9$ for $r=2$ and 20, from Ref. [38] and $L=2-8$ for $r=10$, this work, where the data analysis is more refined (see Appendix A) leading to error bars 10 times smaller). 
The convergence of effective scaling dimensions at different strip sizes, obtained with a cutoff value in the range $\epsilon=10^{-4}-10^{-6}$ and $r=r^{\star}(Q)$, is shown in Fig. 7 for different values of $Q$. The extrapolation in the thermodynamic limit is given in Table III. The details of the fitting procedure and of the evaluation of errors is presented in Appendix A.

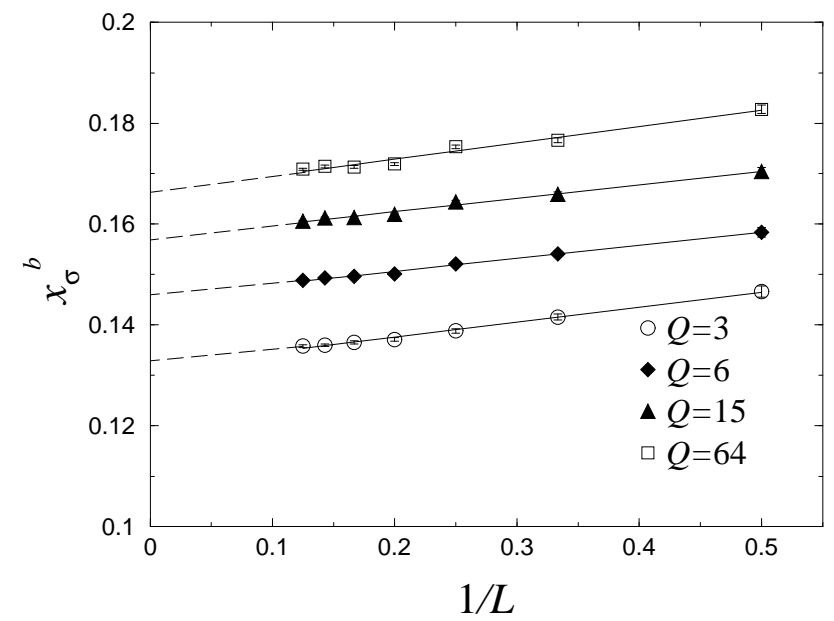

FIG. 7. Magnetic scaling index deduced from the algebraic decay of the average correlation function along the strip of size $L$ as a function of $L^{-1}$ and extrapolation in the thermodynamic limit for different $Q$-values $(L=2-8)$.

TABLE III. Bulk magnetic scaling index (after extrapolation in the thermodynamic limit) obtained from the decay of the correlation function along the strip (cutoff parameter $\left.\epsilon=10^{-4}-10^{-6}\right)$.

\begin{tabular}{llll}
\hline \hline$Q$ & $r$ & $x_{\sigma}^{b}$ & $\Delta x_{\sigma}^{b}$ \\
\hline 3 & 5 & 0.1321 & $3 \times 10^{-4}$ \\
4 & 7 & 0.1385 & $3 \times 10^{-4}$ \\
5 & 7 & 0.1423 & $3 \times 10^{-4}$ \\
6 & 8 & 0.1456 & $3 \times 10^{-4}$ \\
8 & 10 & 0.1505 & $3 \times 10^{-4}$ \\
15 & 10 & 0.1572 & $3 \times 10^{-4}$ \\
64 & 12 & 0.1669 & $3 \times 10^{-4}$ \\
\hline \hline
\end{tabular}

\section{SQUARE GEOMETRY AND CRITICAL BEHAVIOR}

\section{A. Conformal rescaling of boundary effects}

Monte Carlo simulations of two-dimensional spin systems are generally performed on systems of square shape. In the following, we consider such a system of size $N \times N$, and call $u$ and $v$ the corresponding directions (Fig. 8). a)

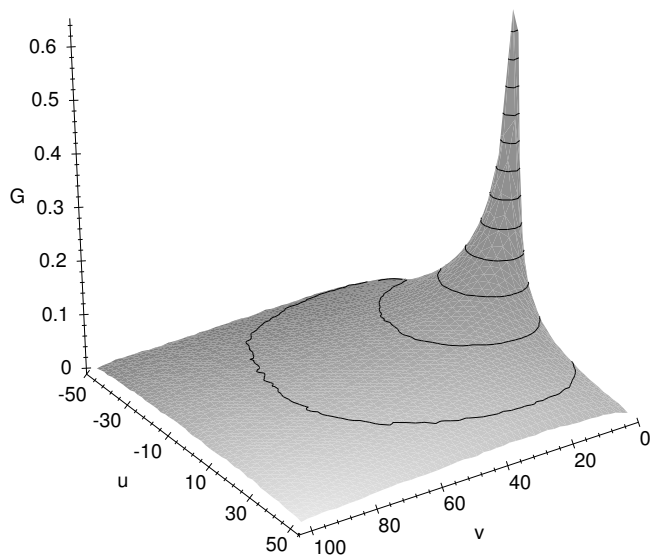

b)

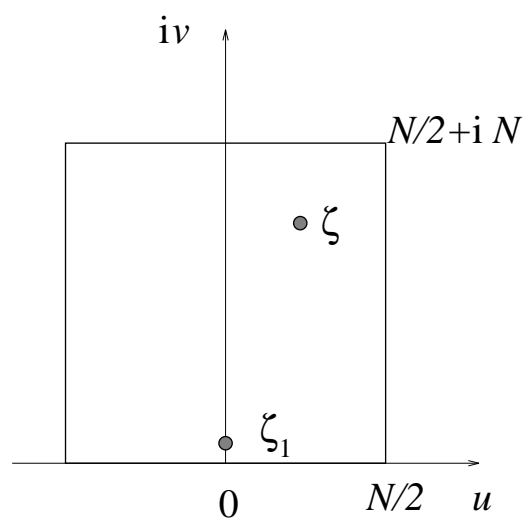

FIG. 8. a) Monte Carlo simulations of the $2 d$ RBPM inside a square of $101 \times 101$ lattice sites $\left(10^{6} \mathrm{MCS} / \mathrm{spin}\right.$, Swendsen-Wang cluster algorithm). The figure shows the correlation function between a point close to the surface $\left(\zeta_{1}=\mathrm{i}\right)$ and all other points $\zeta$ in the square. The notations are specified in b).

The order parameter correlation function between a point close to the surface, and a point in the bulk of the system should, in principle, lead to both surface and bulk critical exponents, possibly to structure constants 46. Practically, FSS techniques are not of great help for the accurate determination of critical exponents, since

i) strong surface effects (shape effects) occur which modify the large distance power-law behavior, i.e. the scaling regime, 
ii) the universal scaling function entering the correlation function is likely to display a crossover before its asymptotic regime is reached (system-dependent effect).

One can proceed as follows: Systems of increasing sizes are successively considered, and the correlations are computed along $u$ - (parallel to a square edge considered as the free surface) and $v$-axis (perpendicular to this edge). The order parameter correlation function for example is supposed to obey a scaling form which reproduces the expected power-law behavior in the thermodynamic limit:

$$
\begin{gathered}
G_{\perp}^{\mathrm{sq}}(v)=\frac{1}{v^{x_{\sigma}^{b}+x_{\sigma}^{1}}} f_{\perp}^{\mathrm{sq}}\left(\frac{v}{N}\right), \\
G_{\|}^{\mathrm{sq}}(u)=\frac{1}{u^{2 x_{\sigma}^{1}}} f_{\|}^{\mathrm{sq}}\left(\frac{u}{N}\right),
\end{gathered}
$$

where $x_{\sigma}^{b}$ and $x_{\sigma}^{1}$ are the bulk and surface order parameter scaling dimensions, respectively. The scaling functions have to satisfy asymptotic expansions including corrections to scaling due to the limitations mentioned above, e.g. $f_{\perp}^{\mathrm{sq}}\left(\frac{v}{N}\right) \sim 1+$ const $\times\left(\frac{v}{N}\right)^{\mu}+\ldots$ in the boundary region $v \rightarrow N$.

Equations (16) and (17) are not very useful for the determination of critical exponents, since the scaling regime $v \rightarrow N$ is perturbed by the correction terms which have a large amplitude, resulting from the significance of finitesize corrections. Nevertheless, conformal invariance supplies an easy way to take into account explicitly shape effects in two-dimensional systems, and thus provides a refined procedure for the determination of the exponents. In pure systems, density profiles, correlations and local properties have been investigated in various geometries (surfaces 47 49, corners [50 52], strips 53 55] or parabolic shapes [56 61], for a review, see Ref. [62]), as well as the moments of the magnetization 63 and structure factors 64 have been calculated in square systems.

In the following, we shall consider a square system with free or fixed boundary conditions on all the edges. Using conformal invariance techniques [65], the SchwarzChristoffel mapping enables us to calculate the surfacebulk correlation fonction inside the square. The mapping of the complex half-plane $z=x+\mathrm{i} y, \operatorname{Im} z>0$, inside a square $\zeta=u+\mathrm{i} v,-N / 2 \leq \operatorname{Re} \zeta \leq N / 2,0 \leq \operatorname{Im} \zeta \leq N$, is realized by the conformal transformation 66 .

$$
\frac{\mathrm{d} \zeta}{\mathrm{d} z}=\frac{C}{\sqrt{\left(1-z^{2}\right)\left(1-k^{2} z^{2}\right)}} .
$$

Since $\zeta=N / 2$ and $\zeta=N / 2+\mathrm{i} N$ are mapped onto $z=1$ and $z=1 / k(0<k<1)$, respectively, the constant $C$ is related to the size of the square

$$
\begin{aligned}
N / 2 C & =\mathrm{K}(k) \equiv \mathrm{K}, \\
N / C & =\mathrm{K}\left(k^{\prime}\right) \equiv \mathrm{K}^{\prime},
\end{aligned}
$$

where $k^{\prime}=\sqrt{1-k^{2}}$ and $\mathrm{K}(k)$ is the complete elliptic integral of the first kind. The modulus $k$ also follows from these equations. It is given by 66

$$
k=4\left(\frac{\sum_{p=0}^{\infty} q^{(p+1 / 2)^{2}}}{1+2 \sum_{p=1}^{\infty} q^{p^{2}}}\right)^{2}, \quad q=\mathrm{e}^{-2 \pi} .
$$

The complete transformation is finally written

$$
\begin{aligned}
& \zeta=\frac{N}{2 \mathrm{~K}} \mathrm{~F}(z, k)=\frac{N}{\mathrm{~K}^{\prime}} \mathrm{F}(z, k), \\
& z=\operatorname{sn} \frac{\mathrm{K}^{\prime} \zeta}{N} \equiv \operatorname{sn}\left(\frac{\mathrm{K}^{\prime} \zeta}{N}, k\right),
\end{aligned}
$$

where $\mathrm{F}(z, k)$ is the elliptic integral of the first kind and $\operatorname{sn}(\zeta, k)$ the Jacobian elliptic sine [67].

\section{B. Correlation functions}

The two-point correlation function of a conformally invariant system can now be obtained in the $\zeta$-geometry in terms of its counterpart in the semi-infinite system (z-geometry):

$$
\begin{aligned}
G\left(\zeta_{1}, \zeta\right) & \equiv\left\langle\sigma\left(\zeta_{1}\right) \sigma(\zeta)\right\rangle_{\mathrm{sq}} \\
& =\left|\zeta^{\prime}\left(z_{1}\right)\right|^{-x_{\sigma}^{b}}\left|\zeta^{\prime}(z)\right|^{-x_{\sigma}^{b}}\left\langle\sigma\left(z_{1}\right) \sigma(z)\right\rangle_{\mathrm{hp}},
\end{aligned}
$$

where the correlation function in the half-plane (hp) geometry is known to take the form 47

$$
\begin{aligned}
G\left(z_{1}, z\right) & \equiv\left\langle\sigma\left(z_{1}\right) \sigma(z)\right\rangle_{\mathrm{hp}} \\
& =\text { const } \times\left(y_{1} y\right)^{-x_{\sigma}^{b}} \psi(\omega),
\end{aligned}
$$

where the dependence on $\omega=\frac{y_{1} y}{\left|z_{1}-z\right|^{2}}$ of the universal scaling function $\psi$ is constrained by the special conformal transformation and its asymptotic behavior, $\psi(\omega) \sim \omega^{x_{\sigma}^{1}}$, in the limit $y_{1}=\mathrm{O}(1), y \gg 1$, is implied by scaling.

Equations. (24) and (23), applied in the random situation, lead to the correlations between $\zeta_{1}=\mathrm{i}$, close to a side of the square, and any point inside it, as follows:

$$
\left[\left\langle G_{\sigma}(\zeta)\right\rangle\right]_{\mathrm{av}}=\text { const } \times\left\{\left|\zeta^{\prime}(z)\right| \operatorname{Im}(z(\zeta))\right\}^{-x_{\sigma}^{b}} \psi(\omega) \text {. }
$$

Taking the logarithm of both sides, the bulk critical exponent $x_{\sigma}^{b}$ can thus be deduced from a linear fit along $\omega=$ const curves in the square:

$$
\ln \left[\left\langle G_{\sigma}(\zeta)\right\rangle\right]_{\mathrm{av}}=\text { const }^{\prime}-x_{\sigma}^{b} \ln \kappa(\zeta)+\ln \psi(\omega),
$$

with

$$
\kappa(\zeta) \equiv \operatorname{Im}(z(\zeta))\left|\left[1-z^{2}(\zeta)\right]\left[1-k^{2} z^{2}(\zeta)\right]\right|^{-1 / 2} .
$$

We will now discuss the results of MC simulations performed with the Swendsen-Wang cluster algorithm 68 for systems of size $101 \times 101$ with canonical disorder. The details concerning the choice of the parameters for the simulations (number of $\mathrm{MC}$ iterations, ... ) are given 
in Appendix B. Average over disorder is performed over $N_{\text {rdm }}=3000$ samples. All the MC simulations are done at the optimal disorder amplitude $r^{\star}(Q)$ determined in the strip geometry.

Eq. (26) is used in Fig. 9 to extract the bulk magnetization scaling dimension at $Q=8$. Consistent values are obtained for different fixed values of the parameter $\omega$. Averaging the results at different $\omega$ 's, one obtains

$$
x_{\sigma}^{b}(8)=0.152 \pm 0.003
$$

corresponding to an error of $2 \%$.
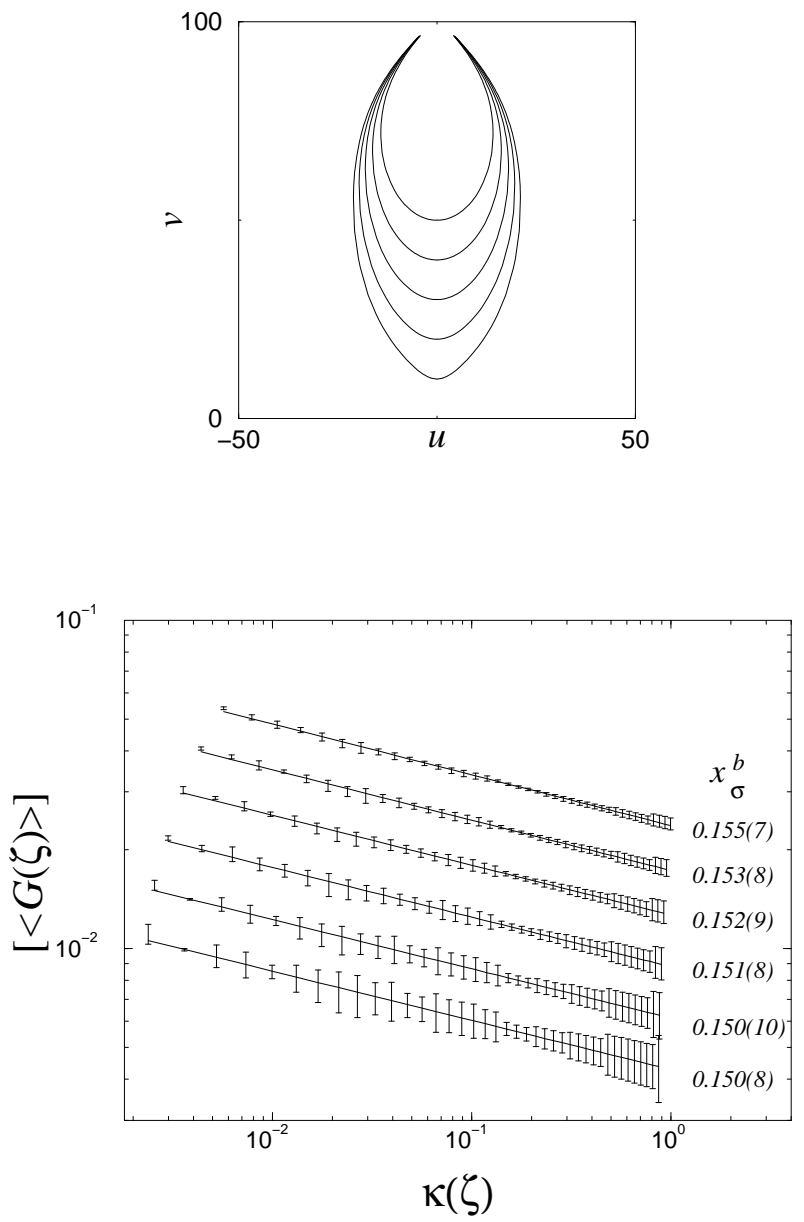

FIG. 9. Rescaled correlation function along six $\omega=$ const curves in the square (shown in the upper part). These curves are approximated by linear expansions in the neighborhood of the discrete lattice sites, which explains the variations on the sizes of error bars $(Q=8, r=10)$.

One should nevertheless mention that the uncertainty on this result is underestimated, since neither the fluctuations due to randomness, nor the influence of a variation of $r$ around the optimal value has been taken into account explicitly. This is intentional, since such studies would require intensive computational efforts and would be less accurate that the next method to be presented.

\section{Density profiles}

Owing to the unknown scaling function $\psi(\omega)$, the determination of the bulk critical exponent from the behavior of the correlation function is not extremely accurate. Furthemore, since a few points are used for the fits along $\omega=$ const. curves, this introduces a poor statistics. It can nevertheless be improved if one considers the magnetization profile inside a square with fixed boundary conditions. Since it is a one-point function, its decay from the distance to the surface in the semi-infinite geometry is fixed, up to a constant prefactor

$$
\left[\langle\sigma(z)\rangle_{\mathrm{hp}}\right]_{\mathrm{av}} \sim y^{-x_{\sigma}^{b}} .
$$

The local order parameter is defined, according to Ref. 69], as the probability for the spin at site $\zeta$ in the square, to belong to the majority orientation (Fig. 10).

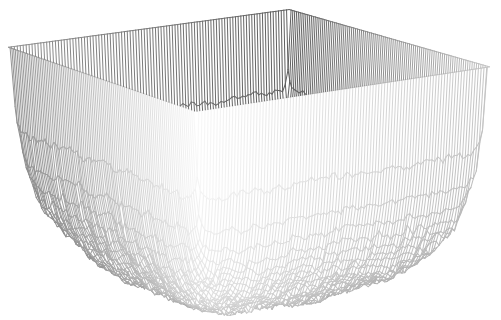

FIG. 10. Density profiles inside the square averaged for 3000 disorder realizations $(Q=8, r=10)$.

The Schwarz-Christoffel mapping leads to the following expression for the average profile in the square geometry:

$$
[\langle\sigma(\zeta)\rangle]_{\mathrm{av}}=\mathrm{const} \times\left(\frac{\sqrt{\left|1-z^{2}(\zeta)\right| \cdot\left|1-k^{2} z^{2}(\zeta)\right|}}{\operatorname{Im}(z(\zeta))}\right)^{x_{\sigma}^{b}}
$$

This expression, of the form $[\langle\sigma(\zeta)\rangle]_{\mathrm{av}}=[f(z) / y]^{x_{\sigma}^{b}}$, holds for any point inside the square. It allows an accurate determination of the critical exponent, since the $N^{2}$ lattice points enter the power-law fit (Fig. 11). Although this technique is more precise than the previous one, one has to take care to different sources of error. It is indeed again necessary to consider the influence of the number of disorder configurations which are used to get the average magnetization, as well as the effect of a variation of the disorder amplitude around the optimal value. We performed $N_{\text {rdm }}=5000$ realizations of disorder in five independent runs (see Appendix B), and computed the magnetic exponent for each run. Averaging the results, it yields the values given in Table IV. 


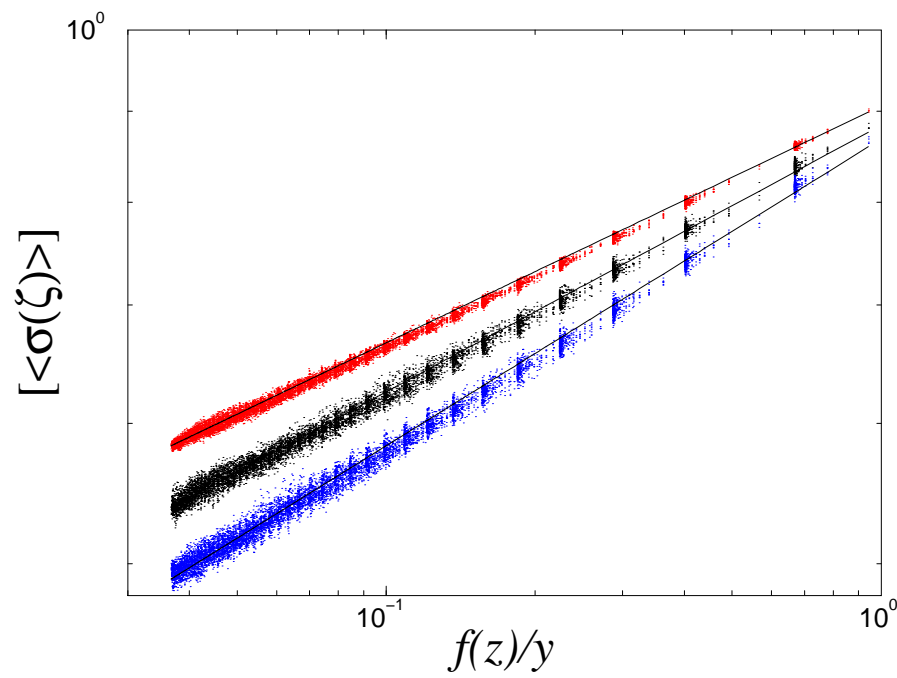

FIG. 11. Rescaled magnetization profile inside the square for 5000 disorder realizations $(Q=3,8$ and 64 from top to bottom). The power law fits are over $100^{2}$ data points.

TABLE IV. Bulk magnetic scaling index obtained from the magnetization profile inside the square (5000 realizations of disorder).

\begin{tabular}{llll}
\hline \hline$Q$ & $r$ & $x_{\sigma}^{b}$ & $\Delta x_{\sigma}^{b}$ \\
\hline 3 & 5 & 0.13357 & $3 \times 10^{-5}$ \\
4 & 7 & 0.13815 & $4 \times 10^{-5}$ \\
5 & 7 & 0.14302 & $4 \times 10^{-5}$ \\
6 & 8 & 0.14621 & $5 \times 10^{-5}$ \\
8 & 10 & 0.15031 & $5 \times 10^{-5}$ \\
15 & 10 & 0.15984 & $6 \times 10^{-5}$ \\
64 & 12 & 0.17299 & $6 \times 10^{-5}$ \\
\hline \hline
\end{tabular}

\section{Boundary critical behavior}

The surface scaling dimension can be obtained once the bulk exponent is known. From standard scaling, the asymptotic behavior of the two-point correlation function, when $y_{1}=\mathrm{O}(1), y \gg 1$ is expected to involve both bulk and surface dimensions:

$$
\left[\left\langle G_{\sigma}\left(y-y_{1}\right)\right\rangle\right]_{\mathrm{av}} \sim y^{-\left(x_{\sigma}^{b}+x_{\sigma}^{1}\right)} .
$$

A power law behavior thus follows for the universal scaling function defined in Eq. (24):

$$
\begin{aligned}
\psi(\omega) & \equiv\left[\left\langle G_{\sigma}(\zeta)\right\rangle\right]_{\mathrm{av}} \times\left\{\left|\zeta^{\prime}(z)\right| \operatorname{Im}(z(\zeta))\right\}^{x_{\sigma}^{b}} \\
& \sim \omega^{x_{\sigma}^{1}}, \quad \omega \rightarrow 0 .
\end{aligned}
$$

A log-log plot of Eq. (32) is shown on Fig. 12, where the TM results are also presented for comparison. The result for the surface scaling index is less accurate than in the case of the bulk, but the estimation $x_{\sigma}^{1}(8) \simeq 0.47(3)$ is in agreement with the value that we obtained previously by FSS techniques in Ref. [22]. It also agrees with the TM results which give $x_{\sigma}^{1}(8) \simeq 0.48(2)$ for $L=7$ and $x_{\sigma}^{1}(8) \simeq 0.50(2)$ for $L=8$.

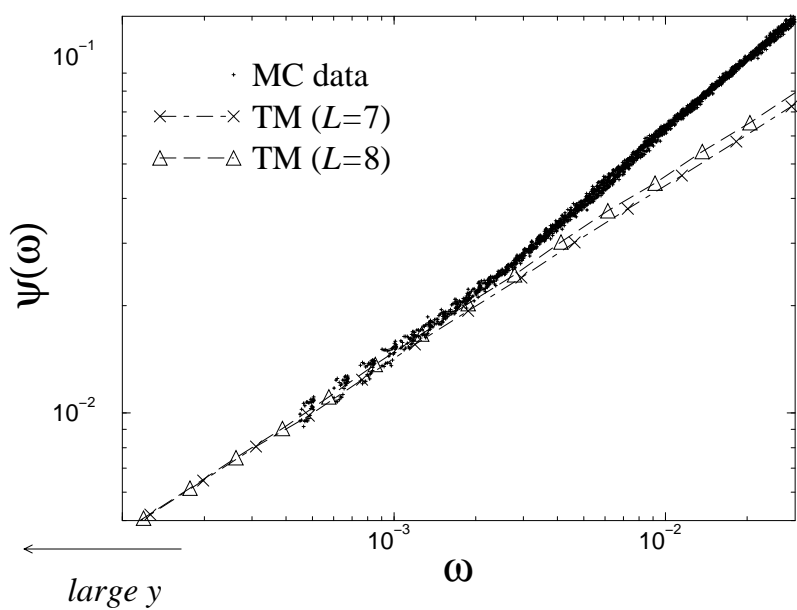

FIG. 12. Large distance behavior of the universal scaling function $(Q=8, r=10)$ leading to the surface scaling index. The fit has been shifted for clarity.

If the leading singularity $\left(x_{\sigma}^{1}\right)$ is found to be the same using the two techniques, we note that the corrections to scaling are very different, as it appears in the deviation between the curves as $\omega$ increases. This can be the result of the ensemble average procedure which is not identical in the two approaches (grand canonical for the TM technique and canonical disorder for the MC simulations). The same type of sensitivity to the ensemble average was reported recently by Wiseman and Domany [41].

\section{CONCLUSION}

In this paper, we have investigated the magnetic critical properties of disordered $Q$-state Potts ferromagnets for a wide range of $Q$-values. These models lead to second-order phase transitions which are particularly interesting, since they belong to new universality classes. The accurate determination of critical indices is a preliminary step towards a deeper understanding of these universality classes. Although universality is expected with respect to the disorder amplitude $r$, previous works on finite systems have shown that the numerical results are very sensitive to the choice of this disorder amplitude. This sensitivity is attributed to crossover effects due to the pure model $(r \rightarrow 1)$ and percolation $(r \rightarrow \infty)$ unstable fixed points. The behavior of the effective central charge as a function of $r$ can fortunately be exploited to locate the optimal regime of disorder. One should mention that in our previous studies, this extreme sensitivity of the numerical estimates of critical exponents 
was not well understood, resulting in an underestimation of uncertainties. We tried to present here a carefull analysis leading to reliable error bars. This uncertainty is mainly due to the non self-averaging behavior of correlation functions. In the strip geometry, the number of samples being already important, better estimates would not be easy to obtain, whilst in the MC simulations, improvements could be supplied by increasing the number of realizations of disorder.

The conformal mapping inside the square seems very efficient compared to standard FSS studies, one lattice size being needed only. The accuracy is furthermore substantially improved, since

i) the finite-size corrections are essentially included in the conformal mapping, files.

ii) all the lattice points enter the fit of the density pro-

A summary of our results, compared to other independent determinations of the magnetic scaling index, is given in Table $\mathrm{V}$, and the dependence on $Q$ is shown in Fig. 13. The pure model value for $Q \leq 4$ is shown for comparison 70]. Both FSS and conformal invariance results are presented. The two techniques used in this work are in agreement with each other, as well as with previous studies at the same disorder amplitude, at least as long as the number of states $Q$ is not too large. When the ratio $r$ is very different, disagreement with other studies which are likely due to crossover effects occurs. On the other hand, when the number of states is large, $Q>15$, there appears discrepancies between the two techniques used here. Whilst the second method (square geometry, 5000 realizations) seems to be the most accurate, we are more confident in the first one (strip geometry, 80000 realizations): If the number of disorder realisations is too small, the average behaviour will indeed give an exponent closer to the typical one, and thus too large. MC simulations are furthermore known to be less efficient when $Q$ increases, since the autocorrelation time increases also, requiring larger numbers of thermalisation iterations.

We also note that the leading singularity of the magnetization does not depend, up to the precision of our results, on the type of disorder considered (GCD or CD).

TABLE V. Extrapolation of the bulk magnetic scaling dimension $x_{\sigma}^{b}$ in the thermodynamic limit for the different values of $Q$. The first two columns recall previous FSS results obtained by MC simulations (in which the accuracy had been overestimated, since the influence of the disorder amplitude was not well understood, at least in which concerns our own studies). The data in the four remaining columns were deduced from conformal invariance. The quantity that was studied is indicated in the table as well as the geometry and the numerical technique. The results presented in this work are written in bold face. The table notes recall the parameters used for each result, especially the values of disorder amplitude which are known to have strong influence on the exponent.

\begin{tabular}{|c|c|c|c|c|c|c|}
\hline \multirow[b]{2}{*}{$Q$} & \multicolumn{2}{|c|}{$\begin{array}{l}\text { FSS(MC) } \\
\text { square }\end{array}$} & \multicolumn{4}{|c|}{ Conformal Invariance } \\
\hline & $\begin{array}{l}\mathrm{SW} \\
{\left[\left\langle M_{b}\right\rangle\right]}\end{array}$ & $\begin{array}{l}\mathrm{W} \\
{\left[\left\langle M_{b}\right\rangle\right]}\end{array}$ & $\begin{array}{l}\text { TM } \\
{[\langle G(u)\rangle]}\end{array}$ & $\begin{array}{l}\text { TM } \\
{[\langle G(u)\rangle]}\end{array}$ & $\begin{array}{l}\mathrm{SW} \\
{[\langle G(\zeta)\rangle]}\end{array}$ & $\begin{array}{l}\mathrm{SW} \\
{[\langle\sigma(\zeta)\rangle]}\end{array}$ \\
\hline 4 & $0.145(3)^{\mathrm{e}}$ & $0.139^{\mathrm{f}}$ & $0.1396(5)^{\mathrm{b}}$ & $0.1385(3)^{\mathrm{C}}$ & & $0.13815(4)^{\mathrm{d}}$ \\
\hline 5 & & & $0.1413(10)^{\mathrm{b}}$ & $0.1423(3)^{c}$ & & $0.14302(4)^{d}$ \\
\hline 8 & $0.153(3)^{\mathrm{i}}$ & $0.151(4)^{\mathrm{h}}$ & $0.1496(9)^{\mathrm{j}}$ & $0.1505(3)^{\mathrm{c}}$ & $0.152(3)^{\mathrm{k}}$ & $0.15031(5)^{\mathrm{d}}$ \\
\hline 15 & & & & $0.1572(3)^{\mathrm{c}}$ & & $0.15984(6)^{d}$ \\
\hline 64 & & $0.185(5)^{\mathrm{h}}$ & & $0.1669(3)^{c}$ & & $0.17299(6)^{d}$ \\
\hline
\end{tabular}

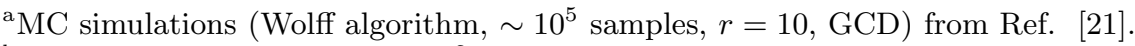

${ }^{\mathrm{b}} \mathrm{TM}$ calculations $\left(L=1-7,10^{2}\right.$ samples, $r=2$, GCD) from Refs. [17, 18].

${ }^{\mathrm{c}} \mathrm{TM}$ calculations $\left(L=2-8,80 \times 10^{3}\right.$ samples, the values of disorder amplitude for $Q=3,4,5,6,8,15$ and 64 are $r=5,7$, $7,8,10,10$ and 12 , respectively, GCD), this work.

${ }^{\mathrm{d}} \mathrm{MC}$ simulations (Swendsen-Wang algorithm, $N=101,5 \times 10^{3}$ samples, the values of disorder amplitude for $Q=3,4,5,6,8$, 15 and 64 are $r=5,7,7,8,10,10$ and 12, respectively, CD), this work.

${ }^{\mathrm{e}} \mathrm{MC}$ simulations (cluster algorithm, $N=256, \sim 500$ samples, $r=10$, GCD) from Ref. [15].

${ }^{\mathrm{f}} \mathrm{MC}$ simulations (Wolff algorithm) M. Picco, Ref. [52] cited in Ref. [18].

${ }^{\mathrm{g}} \mathrm{MC}$ simulations (Swendsen-Wang algorithm, $N \leq 100, \sim 30$ samples, $r=2$, restricted CD) from Ref. 12 .

${ }^{\mathrm{h}} \mathrm{MC}$ simulations (Wolff algorithm, $N \leq 100$ and 500, $\sim 10^{5}$ samples, $r=10$, GCD) from Ref. 24.

${ }^{\mathrm{i}} \mathrm{MC}$ simulations (Swendsen-Wang algorithm, $N \leq 100, \sim 500$ samples, $r=10, \mathrm{CD}$ ) from Ref. 22 .

${ }^{\mathrm{j}} \mathrm{TM}$ calculations $\left(L=2-9,40 \times 10^{3}\right.$ samples, $r=10$, GCD) from Ref. 38 .

${ }^{\mathrm{k}} \mathrm{MC}$ simulations (Swendsen-Wang algorithm, $N=101,3 \times 10^{3}$ samples, $r=10$, CD) from Ref. 38. 


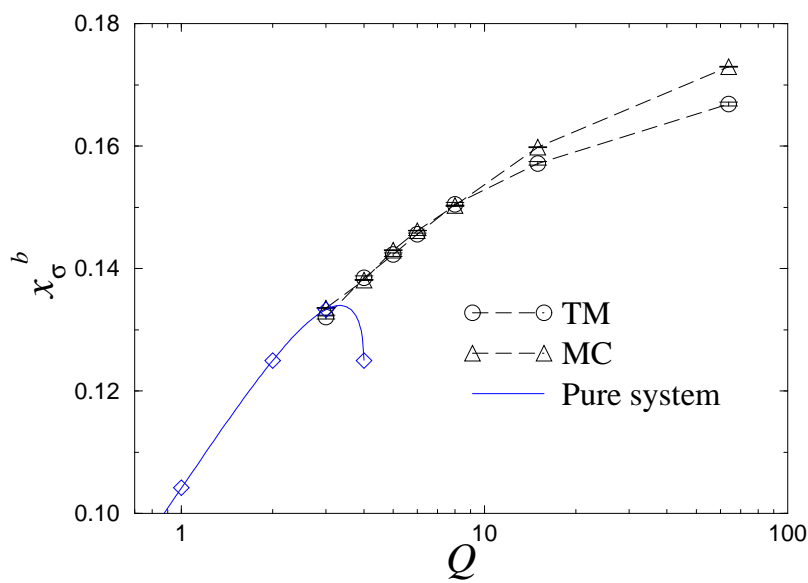

FIG. 13. $Q$-dependence of the bulk magnetic scaling dimension in the RBPM compared to the pure model value for $Q \leq 4$.

In the case $Q=3$ which was already considered by different authors, there exists a perturbative result (renormalization group approach for the perturbative series around the pure model conformal field theory):

$$
x_{\sigma}^{b}=\frac{2}{15}+0.00132 \simeq 0.13465
$$

This result was confirmed numerically by Picco [21] and Cardy and Jacobsen [17]. In this work, we obtain a value which is slightly too small. We nevertheless note that the two values, at $r=5$ and $r=6$ are in perfect agreement (see Appendix B).

We eventually mention a recent work of Olson and Young [71] who performed a MC study of the multiscaling properties of the correlation functions for different values of $Q$. They used a different self-dual probability distribution of the couplings, and obtained slightly different results (e.g. $x_{\sigma}^{b}=0.161(3)$ at $Q=8$ ).

\section{ACKNOWLEDGMENTS}

We thank L. Turban for critically reading the manuscript and J.L. Jacobsen for suggesting us to control the stability of the exponents around the optimal disorder amplitude. We are also indebted to M. Picco who drew our attention on refined analysis of the data which reduces the error bars.

Due to the disorder average, the numerical study of disordered systems is particularly suitable to parallel computing. The computations presented here were performed on the SP2 at the CNUSC in Montpellier under projects No. C981009 and C990011, and the Power Challenge Array at the CCH in Nancy. The Laboratoire de Physique des Matériaux is Unité Mixte de Recherche CNRS No. 7556 .

\section{APPENDIX A: EVALUATION OF ERRORS IN THE TRANSFER MATRIX CALCULATIONS}

In spite of the large number of disorder realizations, the correlation functions along the strip display an important dispersion but the resulting values for the critical exponents are extremely accurate. In order to obtain a correct estimation of the errors on the magnetic scaling index, we studied the influence of the cutoff parameter $\epsilon$. For $\epsilon \simeq 10^{-1}$, a few points are taken into account only and the short distance behavior of the correlation func- tion is observed. On the other hand, with $\epsilon \simeq 10^{-6}$, all the data points in the range $u=5-100$ are taken into account in the fit, giving a greater weight to the longdistance behavior. Clearly, one has to find a compromise between the two approaches. Fortunately a variation of the cutoff parameter does not affect the value of the extrapolated exponent which remains very stable as shown in Table VI. 
TABLE VI. Bulk magnetic scaling index (after extrapolation in the thermodynamic limit) obtained from the decay of the correlation function along the strip with different values of the cutoff $\epsilon$.

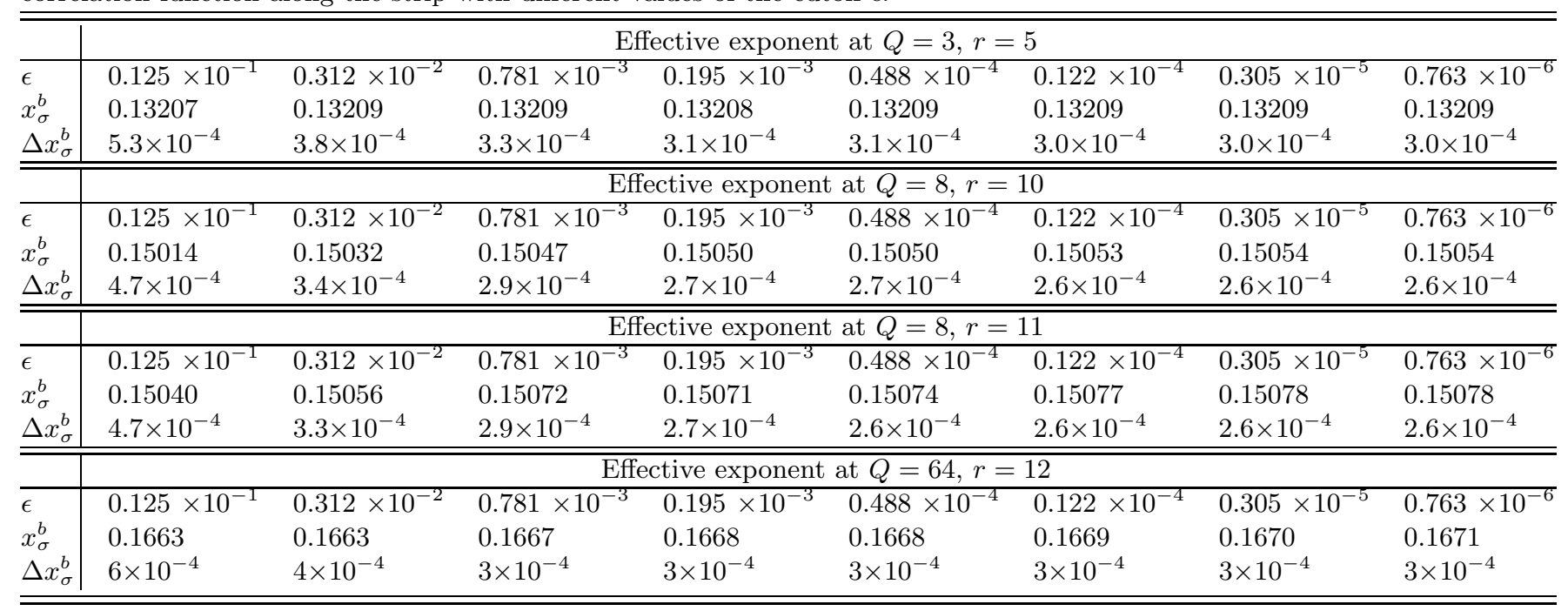

Another contribution to the error should come from the choice of the disorder amplitude. To study this effect, we considered a variation of $r$ close to the optimal value. It leads to a result which is inside the error bars of the previous one, as shown in the case $Q=8$ in Table VI. The uncertainty in the range $\epsilon=10^{-4}-10^{-6}$ is of the same order of magnitude than the fluctuations between the data obtained with different values of $\epsilon$ and $r$, so we eventually consider as a definitive result the fit with this cutoff value.

\section{APPENDIX B: DETAILS OF THE MONTE CARLO SIMULATIONS}

In random systems, in addition to the usual MC error, the random-bond fluctuations introduce another source of statistical error. For any physical quantity $X$, the total error is given by

$$
(\delta X)^{2}=\frac{\sigma_{\mathrm{rdm}}^{2}}{N_{\mathrm{rdm}}}+\frac{\sigma_{T}^{2}\left(1+2 \tau_{X}\right)}{N_{\mathrm{rdm}} N_{\mathrm{MC}}}
$$

where the first term is due to the disorder fluctuations, whilst the second one describes the fluctuations during the MC iterations. This latter term corresponds to the standard deviation of independent random variables, corrected by the autocorrelation time to take into account the correlations between the successive data. In these expressions, $N_{\mathrm{MC}}$ is the number of $\mathrm{MC}$ iterations, measured in MC steps (MCS), realized for the measurements of the physical quantities for each disorder realization, $N_{\mathrm{rdm}}$ is the number of disorder realizations and $\tau_{X}$ is the autocorrelation time for the quantity $X$ (the definition of $\tau_{X}$ sometimes absorbs the factor 1 describing uncorrelated variables). The variances $\sigma_{T}$ and $\sigma_{\text {rdm }}$ respectively measure the deviation due to thermal fluctuations for a given sample and the deviation from the exact value within the ensemble of disorder configurations.

Both variances are of the same order of magnitude. The leading source of error thus comes from the disorder average and a large number of samples is needed in order to get accurate results. In our simulations we used the Swendsen-Wang cluster algorithm 68 for systems of size $101 \times 101$. The autocorrelation time for the total magnetization is $\tau_{\sigma} \simeq 35 \mathrm{MCS}$. The preliminary $5000 \mathrm{MCS}$ have been discarded for thermalisation (better that $10^{2} \times \tau_{\sigma}$ ), and $N_{\mathrm{MC}}=10^{4}$ MCS were done to compute the physical quantities. Average over disorder is performed over $N_{\text {rdm }}=5000$ samples. From preliminary runs over 1000 samples, we deduced the standard deviations $\sigma_{\text {rdm }}^{2} \simeq 0.93$ and $\sigma_{\mathrm{MC}}^{2} \simeq 0.13$. The order of magnitude of the two contributions to the error is thus

$$
\begin{aligned}
& \delta \sigma_{\mathrm{MC}} \simeq \sqrt{\frac{\sigma_{T}^{2}\left(1+2 \tau_{\sigma}\right)}{N_{\mathrm{rdm}} N_{\mathrm{MC}}}} \simeq 6 \times 10^{-4}, \\
& \delta \sigma_{\mathrm{rdm}} \simeq \sqrt{\frac{\sigma_{\mathrm{rdm}}^{2}}{N_{\mathrm{rdm}}}} \simeq 1.36 \times 10^{-2},
\end{aligned}
$$

for a point in the middle of the square. Due to the fixed boundary conditions, close to the edges of the square the fluctuations are reduced. These values confirm the significance of the disorder contribution $\left(\frac{\delta \sigma_{\mathrm{MC}}}{[\langle\sigma\rangle]_{\mathrm{av}}} \simeq 0.08 \%\right.$ and $\left.\frac{\delta \sigma_{\mathrm{rdm}}}{[\langle\sigma\rangle]_{\mathrm{av}}} \simeq 2 \%\right)$. The values of the exponent $x_{\sigma}^{b}$ for different values of $Q$ and $r$ are given in Table VII. 
TABLE VII. Bulk magnetic scaling index obtained from the profile of the order parameter inside a square with fixed boundary conditions for five independent runs (1000 configurations of disorder for each run). The final result obtained with 5000 configurations of disorder is given in the column called average.

\begin{tabular}{|c|c|c|c|c|c|c|}
\hline & \multicolumn{6}{|c|}{ Exponent at $Q=3, r=5$} \\
\hline & run 1 & run 2 & run 3 & run 4 & run 5 & average \\
\hline$x_{\sigma}^{b}$ & 0.13260 & 0.13384 & 0.13405 & 0.13418 & 0.13323 & 0.13357 \\
\hline \multirow[t]{2}{*}{$\Delta x_{\sigma}^{b}$} & $7 \times 10^{-5}$ & $7 \times 10^{-5}$ & $7 \times 10^{-5}$ & $7 \times 10^{-5}$ & $7 \times 10^{-5}$ & $3 \times 10^{-5}$ \\
\hline & \multicolumn{6}{|c|}{ Exponent at $Q=3, r=6$} \\
\hline \multirow{4}{*}{$\begin{array}{l}x_{\sigma}^{b} \\
\Delta x_{\sigma}^{b} \\
\end{array}$} & run 1 & run 2 & run 3 & run 4 & run 5 & average \\
\hline & 0.13450 & 0.13262 & 0.13307 & 0.13378 & 0.13333 & 0.13345 \\
\hline & $7 \times 10^{-5}$ & $7 \times 10^{-5}$ & $7 \times 10^{-5}$ & $7 \times 10^{-5}$ & $7 \times 10^{-5}$ & $3 \times 10^{-5}$ \\
\hline & \multicolumn{6}{|c|}{ Exponent at $Q=4, r=7$} \\
\hline \multirow{4}{*}{$\begin{array}{l}x_{\sigma}^{b} \\
\Delta x_{\sigma}^{b}\end{array}$} & run 1 & run 2 & run 3 & $\operatorname{run} 4$ & run 5 & average \\
\hline & 0.13886 & 0.13835 & 0.13798 & 0.13703 & 0.13858 & 0.13815 \\
\hline & $8 \times 10^{-5}$ & $8 \times 10^{-5}$ & $8 \times 10^{-5}$ & $8 \times 10^{-5}$ & $8 \times 10^{-5}$ & $4 \times 10^{-5}$ \\
\hline & \multicolumn{6}{|c|}{ Exponent at $Q=4, r=8$} \\
\hline & run 1 & run 2 & run 3 & run 4 & run 5 & average \\
\hline$x_{\sigma}^{b}$ & 0.13799 & 0.13794 & 0.13753 & 0.13849 & 0.13798 & 0.1379 \\
\hline \multirow[t]{2}{*}{$\Delta x_{\sigma}^{b}$} & $9 \times 10^{-5}$ & $9 \times 10^{-5}$ & $9 \times 10^{-5}$ & $9 \times 10^{-5}$ & $9 \times 10^{-5}$ & $4 \times 10^{-5}$ \\
\hline & \multicolumn{6}{|c|}{ Exponent at $Q=8, r=10$} \\
\hline & run 1 & run 2 & run 3 & run 4 & run 5 & average \\
\hline$x_{\sigma}^{b}$ & 0.1508 & 0.1515 & 0.1501 & 0.1501 & 0.1492 & 0.15031 \\
\hline \multirow[t]{2}{*}{$\Delta x_{\sigma}^{b}$} & $1 \times 10^{-4}$ & $1 \times 10^{-4}$ & $1 \times 10^{-4}$ & $1 \times 10^{-4}$ & $1 \times 10^{-4}$ & $5 \times 10^{-5}$ \\
\hline & \multicolumn{6}{|c|}{ Exponent at $Q=8, r=20$} \\
\hline & run 1 & run 2 & run 3 & & & average \\
\hline$x_{\sigma}^{b}$ & 0.14527 & 0.14506 & 0.14505 & & & 0.14513 \\
\hline \multirow[t]{2}{*}{$\Delta x_{\sigma}^{b}$} & $6 \times 10^{-5}$ & $6 \times 10^{-5}$ & $6 \times 10^{-5}$ & & & $3 \times 10^{-5}$ \\
\hline & \multicolumn{6}{|c|}{ Exponent at $Q=64, r=12$} \\
\hline & run 1 & run 2 & run 3 & run 4 & run 5 & average \\
\hline$x_{\sigma}^{b}$ & 0.1722 & 0.1733 & 0.1724 & 0.1747 & 0.1725 & 0.17299 \\
\hline$\Delta x_{\sigma}^{b}$ & $1 \times 10^{-4}$ & $1 \times 10^{-4}$ & $1 \times 10^{-4}$ & $1 \times 10^{-4}$ & $1 \times 10^{-4}$ & $6 \times 10^{-5}$ \\
\hline
\end{tabular}

* To whom all correspondence should be addressed. Electronic address: berche@lps.u-nancy.fr

$\dagger$ The Laboratoire de Physique des Matériaux is Unité Mixte de Recherche C.N.R.S. No. 7556.

[1] L. Schwenger, K. Budde, C. Voges and H. Pfnür, Phys. Rev. Lett. 73, 296 (1994).

[2] C. Voges and H. Pfnür, Phys. Rev. B 57, 3345 (1998).

[3] Ch.V. Mohan, H. Kronmüller and M. Kelsch, Phys. Rev. B 57, 2701 (1998).

[4] W. Selke, L.N. Shchur and A.L. Talapov, in Annual Reviews of Computational Physics, edited by D. Stauffer (World Scientific, Singapore, 1994), Vol 1 p. 17.

[5] A.B. Harris, J. Phys. C 7, 1671 (1974).

[6] B.N. Shalaev, Phys. Rep. 237, 129 (1994)..

[7] Y. Imry and M. Wortis, Phys. Rev. B 19, 3580 (1979).

[8] M. Aizenman and J. Wehr, Phys. Rev. Lett. 62, 2503 (1989).

[9] K. Hui and A.N. Berker, Phys. Rev. Lett. 62, 2507 (1989).
[10] F.Y. Wu, Rev. Mod. Phys. 54, 235 (1982).

[11] S. Chen, A.M. Ferrenberg and D.P. Landau, Phys. Rev. Lett. 69, 1213 (1992).

[12] S. Chen, A.M. Ferrenberg and D.P. Landau, Phys. Rev. E 52, 1377 (1995).

[13] M.A. Novotny and D.P. Landau, Phys. Rev. B 24, 1468 (1981).

[14] M. Kardar, A.L. Stella, G. Sartoni, and B. Derrida, Phys. Rev. E 52, R1269 (1995).

[15] S. Wiseman and E. Domany, Phys. Rev. E 51, 3074 (1995).

[16] J.L. Cardy, J. Phys. A 29, 1897 (1996).

[17] J.L. Cardy and J.L. Jacobsen, Phys. Rev. Lett. 79, 4063 (1997).

[18] J.L. Jacobsen and J.L. Cardy, Nucl. Phys. B 515, 701 (1998).

[19] H.W.J. Blöte and M.P. Nightingale, Physica (Amsterdam) 112A, 405 (1982).

[20] Vl. Dotsenko, M. Picco and P. Pujol, Nucl. Phys. B455 [FS], 701 (1995).

[21] M. Picco, Phys. Rev. B 54, 14930 (1996).

[22] C. Chatelain and B. Berche, Phys. Rev. Lett. 80, 1670 (1998).

[23] F. Yaşar, Y. Gündüç, and T. Çelik, Phys. Rev. E 58, 
4210 (1998).

[24] M. Picco, e-print cond-mat/9802092.

[25] E. Buffenoir and S. Wallon, J. Phys. A: Math. Gen. 26, 3045 (1993).

[26] J.L. Cardy, Physica A 263, 215 (1999).

[27] V.B. Andreichenko, Vl.S. Dotsenko, W. Selke and J.S. Wang, Nucl. Phys. B344, 531 (1990).

[28] J.S. Wang, W. Selke, Vl.S. Dotsenko and V.B. Andreichenko, Physica A 164, 221 (1990).

[29] K. Binder, in Phase Transitions and Critical Phenomena, edited by C. Domb and J.L. Lebowitz (Academic Press, London, 1983), Vol. 8, p. 1.

[30] W. Selke, F. Szalma, P. Lajkó, and F. Iglói, e-print condmat/9707336.

[31] P. Di Francesco, H. Saleur and J.-B. Zuber, Nucl. Phys. B 290 [FS20], 527 (1987).

[32] A.L. Talapov and Vl.S. Dotsenko, e-print condmat/9306027.

[33] A.L. Talapov and L.N. Shchur, Europhys. Lett. 27, 193 (1994).

[34] S.L.A. de Queiroz and R.B. Stinchcombe, Phys. Rev. B 50, 9976 (1994).

[35] S.L.A. de Queiroz, Phys. Rev. E 51, 1030 (1995).

[36] F.D.A. Aarão Reis, S.L.A. de Queiroz and R.R. dos Santos e-print cond-mat/9608083.

[37] M. Picco, Phys. Rev. Lett. 79, 2998 (1997).

[38] C. Chatelain and B. Berche, Phys. Rev. E 58, R6899 (1998).

[39] B. Derrida, Phys. Rep. 103, 29 (1984).

[40] S. Wiseman and E. Domany, Phys. Rev. Lett. 81, 22 (1998).

[41] S. Wiseman and E. Domany, Phys. Rev. E 58, 2938 (1998).

[42] H. Furstenberg, Trans. Am. Math. Soc. 108, 377 (1963).

[43] Vl. Dotsenko, J.L. Jacobsen, M.A. Lewis, and M. Picco, e-print cond-mat/9812227.

[44] A. Crisanti, S. Nicolis, G. Paladin, and A. Vulpiani, J. Phys. A 23, 3083 (1990).

[45] W.H. Press, S.A. Teukolsky, W.T. Vetterling and B.P. Flannery, Numerical Recipes (Cambridge University Press, Cambridge), p. 655.

[46] G.T. Barkema and J. McCabe, J. Stat. Phys. 84, 1067 (1996).
[47] J. L. Cardy, Nucl. Phys. B 240 [FS12], 514 (1984).

[48] G. Gomper and H. Wagner, Z. Phys. B 59, (1985) 193.

[49] T. W. Burkhardt and I. Guim, Phys. Rev. B 36, (1987) 2080.

[50] M. N. Barber, I. Peschel and P.A. Pearce, J. Stat. Phys. 37, (1984) 497.

[51] B. Davies and I. Peschel, J. Phys. A 24, (1991) 1293.

[52] D. Karevski, P. Lajkó and L. Turban, J. Stat. Phys. 86, (1997) 1153.

[53] T. W. Burkhardt and E. Eisenriegler, J. Phys. A 19, (1986) L663.

[54] T. W. Burkhardt and T. Xue, Phys. Rev. Lett. 66, (1991) 895.

[55] L. Turban and F. Iglói, J. Phys. A 30, (1997) L105.

[56] I. Peschel, L. Turban and F. Iglói, J. Phys. A 24, (1991) L1229.

[57] L. Turban and B. Berche, J. Phys. (France) I 3, (1993) 925.

[58] B. Davies and I. Peschel, Ann. Physik 2, (1993) 79.

[59] S. Blawid and I. Peschel, Z. Phys. B 95, (1994) 73.

[60] B. Berche, J.-M. Debierre and H. P. Eckle, Phys. Rev. E 50, (1994) 4542.

[61] C. Kaiser and L. Turban, J. Phys. A 27, (1994) L579.

[62] F. Iglói, I. Peschel and L. Turban, Adv. Phys. 42, (1993) 683.

[63] T.W. Burkhardt and B. Derrida, Phys. Rev. B 32, (1985) 7273 .

[64] P. Kleban, G. Akinci, R. Hemtschke and K.R. Brownstein, J. Phys. A: Math. Gen. 19, (1986) 437.

[65] M. Henkel Conformal invariance and critical phenomena (Springer, Berlin) 1999.

[66] M. Lavrentiev and B. Chabat, Méthodes de la théorie des fonctions d'une variable complexe (Mir, Moscou) (1972) Chap. VII.

[67] M. Abramowitz and I. A. Stegun, Handbook of Mathematical functions (Dover, New-York) (1970) .

[68] R.H. Swendsen and J.S. Wang, Phys. Rev. Lett. 58, 86 (1987).

[69] M.S.S. Challa, D.P. Landau, and K. Binder, Phys. Rev. B 34, 1841 (1986).

[70] R.J. Baxter, Exactly solved models in Statistical Mechanics (Academic Press, London 1982).

[71] T. Olson and A.P. Young, e-print cond-mat/9903068. 